\title{
Streaming algorithms for recognizing nearly well-parenthesized expressions
}

\author{
Andreas Krebs ${ }^{1}$, Nutan Limaye $^{2}$, and Srikanth Srinivasan ${ }^{3 \star}$ \\ 1 University of Tübingen, Germany. mail@krebs-net.de \\ 2 Indian Institute of Technology, Bombay, India. nutan@cse.iitb.ac.in \\ 3 DIMACS Center, Rutgers University, USA. srikanth@dimacs.rutgers.edu
}

\begin{abstract}
We study the streaming complexity of the membership problem of 1-turn-Dyck ${ }_{2}$ and Dyck $_{2}$ when there are a few errors in the input string.

1-turn-Dyck ${ }_{2}$ with errors: We prove that there exists a randomized one-pass algorithm that given $x$ checks whether there exists a string $x^{\prime} \in 1$-turn-Dyck ${ }_{2}$ such that $x$ is obtained by flipping at most $k$ locations of $x^{\prime}$ using:

- $O(k \log n)$ space, $O(k \log n)$ randomness, and poly $(k \log n)$ time per item and with error at most $1 / n^{c}$.

$-O\left(k^{1+\epsilon}+\log n\right)$ space for every $0 \leq \epsilon \leq 1, O(\log n)$ randomness, $O\left(\left(\log { }^{O(1)} n+k^{O(1)}\right)\right)$ time per item, with error at most $1 / 8$.

Here, we also prove that any randomized one-pass algorithm that makes error at most $k / n$ requires at least $\Omega(k \log (n / k))$ space to accept strings which are exactly $k$-away from strings in 1 -turn-Dyck ${ }_{2}$ and to reject strings which are exactly $k+2$-away from strings in 1-turn-Dyck ${ }_{2}$. Since 1-turn-Dyck ${ }_{2}$ and the Hamming Distance problem are closely related we also obtain new upper and lower bounds for this problem.

Dyck $_{2}$ with errors: We prove that there exists a randomized one-pass algorithm that given $x$ checks whether there exists a string $x^{\prime} \in$ Dyck $_{2}$ such that $x$ is obtained from $x^{\prime}$ by changing (in some restricted manner) at most $k$ positions using:

- $O(k \log n+\sqrt{n \log n})$ space, $O(k \log n)$ randomness, poly $(k \log n)$ time per element and with error at most $1 / n^{c}$.

$-O\left(k^{1+\epsilon}+\sqrt{n \log n}\right)$ space for every $0 \leq \epsilon \leq 1, O(\log n)$ randomness, $O\left(\left(\log { }^{O(1)} n+k^{O(1)}\right)\right)$ time per element, with error at most $1 / 8$.
\end{abstract}

\section{Introduction}

The data streaming model was introduced in the seminal work of Alon et al. 3. This model naturally arises in situations where the input data is massive and rereading the input bits is expensive. The main parameters that play a role in designing algorithms in such situations are: the space used by the algorithm, and the number of passes made over the input. An algorithm is said to be an efficient data streaming algorithm, if the space used by the algorithm is substantially lesser than the length of the input (sublinear in the length of the input) and the number of passes is independent of the length of the input. Many variants of this basic model have been studied. (See for example [15] for a survey.)

The membership testing for well-paranthesises strings has been considered in the past. We denote the set of words with balanced parentheses of $l$ different types by Dyck ${ }_{l}$. It is known that there is a $O(\log n)$ space deterministic algorithm for testing membership in Dyck ${ }_{l}$. (In fact the problem is known to be in TC 6].) The problem has been considered from property testing perspective (see for example [2], [17]). Recently, the problem was considered in the streaming model by Magniez et al. 14. It was proved that there is a randomized one-pass streaming algorithm that takes space $O(\sqrt{n \log n})$ and tests membership in Dyck ${ }_{l}$. They also gave an efficient $O\left(\log ^{2} n\right)$ space algorithm which makes bidirectional pass (one forward and one

\footnotetext{
* Supported by NSF grants DMS-0835373
} 
backward pass) on the input. They also proved a lower bound of $\Omega(\sqrt{n})$ for any randomized streaming algorithm that makes a single unidirectional (only forward) pass. Chakrabarti et al. [8] and Jain et al. [12] considered the lower bound problem for unidirectional multi-pass randomized algorithms. In [8] it was proved that any $T$-pass (all passes made in the same direction) randomized algorithm requires $\Omega(\sqrt{n} / T \log \log n)$ space. Whereas [12] proved $\Omega(\sqrt{n} / T)$ space lower bound for the same. In [5] membership testing for other classes of languages was considered. In [4] it was proved that any randomized $T$ pass algorithm (passes made in any direction) for testing membership in a deterministic context-free language requires $\Omega(n / T)$ space.

We consider a slightly general version of the membership testing problem for Dyck . Let $\Sigma_{l}$ denote a set of $l$ pairs of matching parentheses. We say that an opening parenthesis is corrupted if it is replaced by another opening parenthesis. Similarly, a closing parenthesis is corrupted if it is replaced by another closing parenthesis. For a language $L \in \Sigma_{l}^{*}$, let $\Delta^{\leq k}(L)$ be defined as the set of words over $\Sigma_{l}^{*}$ obtained by corrupting at most $k$ indices of any word in $L$. In this paper, we consider the membership problem for $\Delta \leq k$ (Dyck $)_{l}$ and $\Delta^{\leq k}(1$-turn-Dyck $)$, where

$$
\text { 1-turn-Dyck } 2=\left\{w \bar{w}^{R} \mid w \in\left\{\left(,[\}^{n} n \geq 1\right\}\right.\right.
$$

Here, $\bar{w}$ is the string obtained from $w$ by replacing an opening parenthesis by its corresponding closing parenthesis and $w^{R}$ is the reverse of $w$.

Accepting strings with at most $k$ errors is a well-studied problem in many models of computation. In the streaming model, the problem has been studied in the past (see for example Cao et al. [7]). But we believe that the problem needs further investigation; this being the primary goal of this paper.

We observe that the membership testing problem for $\Delta^{\leq k}\left(\right.$ Dyck $\left._{l}\right)\left(\Delta^{\leq k}\left(1\right.\right.$-turn-Dyck $\left.\left.{ }_{l}\right)\right)$ reduces to the membership testing problem of $\Delta^{\leq k}\left(\right.$ Dyck $\left._{2}\right)$ ( $\Delta^{\leq k}$ (1-turn-Dyck $)_{2}$, respectively). We give a simple fingerprinting algorithm for $\Delta^{\leq k}\left(1\right.$-turn-Dyck $\left.{ }_{2}\right)$ that uses $O(k \log n)$ bits of space and randomness. The space requirements of this algorithm are nearly optimal (because of a communication complexity lower bound of [11) but the randomness requirements are not. We consider the question of derandomizing the above. The question of derandomizing streaming algorithms has been considered in the past (see for example [10, [16, ,18, 20]). We show that the algorithm can be modified to work with just $O(\log n)$ bits of randomness, incurring a small penalty in the amount of space used. We then consider similar questions for the more general problem $\Delta \leq k\left(\right.$ Dyck $\left._{2}\right)$. The following table summarizes our algorithmic results:

\begin{tabular}{|c|c|c|c|c|c|}
\hline Problem & $\begin{array}{l}\text { One-pass } \\
\text { Algorithm }\end{array}$ & Space & Randomness & Error & Time (per element) \\
\hline \multirow{3}{*}{ 1-turn-Dyck ${ }_{2}$} & \multirow{3}{*}{$\begin{array}{l}1 \\
2\end{array}$} & $O(k \log n)$ & $O(k \log n)$ & inverse poly & $\operatorname{poly}(k \log n)^{4}$ \\
\hline & & for all $0<\epsilon<1$ : & & & $O\left((\log n) O(1)+l^{2} O(1)\right.$ \\
\hline & & $\frac{O\left(k^{1+\epsilon}+\log n\right)}{O(k \log n+\sqrt{n \ln \sigma} n}$ & $\frac{O(\log n)}{O(k \operatorname{lo} n)}$ & $\mid 1 / 8$ & $\frac{O\left((\log n)^{O(1)}+k^{((1)}\right)}{\operatorname{lol} u(k \log n)^{4}}$ \\
\hline Dyck $_{2}$ & 3 & $\begin{array}{l}\text { for all } 0<\epsilon<1: \\
O\left(k^{1+\epsilon}+\sqrt{n \log n}\right)\end{array}$ & $O(\log n)$ & $1 / 8$ & $O\left((\log n)^{O(1)}+k^{O(1)}\right)$ \\
\hline
\end{tabular}

In all the algorithms in the table above, we assume that the length of the input stream is known.

Using Algorithm 1, we can deduce the number of errors as well as their locations. Using a combination of the algorithm for membership testing of 1-turn-Dyck ${ }_{2}$ due to 14. (which we refer to as MMN algorithm) and Algorithm 1, it is easy to get a membership testing algorithm for $\Delta^{\leq k}\left(\right.$ Dyck $\left._{2}\right)$. However, such an algorithm uses $O(k \sqrt{n \log n})$ space. In order to achieve the claimed bound, we modify their algorithm for testing membership in Dyck ${ }_{2}$ and use that in conjunction with Algorithm 1. In our algorithm, we do not need to store the partial evaluations of polynomials on the stack.

Algorithms 2 and 4 are inspired by the communication complexity protocols of Yao 22] and Huang et al. [11. A mere combination of their ideas, however, is not enough to get the required bounds. The crucial observation here is that Yao's protocol can be derandomized by using a combination of small-bias

\footnotetext{
${ }^{4}$ In the case of $\Delta^{\leq k}(1$-turn-Dyck $)$, this is the exact time per item. However, for $\Delta^{\leq k}\left(\right.$ Dyck $\left._{l}\right)$ it is the time per item on average. In the latter case, the algorithm first reads a block and then uses $O($ poly $(k \log n))$ time per element of the block. Therefore, the time per block is $O(\operatorname{poly}(k \log n) \sqrt{n / \log n})$. Both algorithms use an extra post-processing time of $n^{k+O(1)}$.
} 
distributions and distributions that fool DNF formulae. As this requires very few random bits, we get the desired derandomization. These algorithms are also better as compared to Algorithm 1 and 3 in terms of their time complexity. For Algorithm 2, we first prove that it suffices to give an efficient algorithm for $\mathrm{Ham}_{n, k}$, where $\operatorname{Ham}_{n, k}(x, y)$ for $x, y \in\{0,1\}^{n}$ is 1 if and only if the Hamming distance between $x$ and $y$ is at most $k$.

Finally, we consider the question of optimality. We prove that any algorithm that makes $k / n$ error requires $\Omega(k \log (n / k))$ space to test membership in $\Delta^{\leq k}\left(1\right.$-turn-Dyck $\left.{ }_{2}\right)$ by proving a lower bound on Ham $n, k$. The two problems are related as follow: Let $w \in \Sigma^{2 n}$ and let $w=u v$ where $u \in\left\{\left(,[\}^{n} \text { and } v \in\{),\right]\right\}^{n}$. If ( and ) are both mapped to 0 and [ and ] are both mapped to 1 to obtain $x, y$ from $u, v$ then it is easy to see that $u v \in \Delta^{\leq k}\left(1\right.$-turn-Dyck ${ }_{2}$ ) if and only if $\operatorname{Ham}_{n, k}(x, y)=1$.

The problem $\operatorname{Ham}_{n, k}$ was considered in [22, [11, in simultaneous message model. In [11, a lower bound (in fact, a quantum lower bound) of $\Omega(k)$ was proved for the problem. Their lower bound holds even for constant error protocols. To best of our knowledge no better lower bound is known for the problem. We improve on their lower bound by a $\log (n / k)$ factor under the assumption that the communication protocol is allowed to make small error. Our lower bound can be stated as follows:

Theorem 1. Given two strings $x, y \in\{0,1\}^{n}$ such that either the Hamming distance between $x, y$ is exactly $k$ or exactly $k+2$, any randomized one-pass algorithm that makes error $k / n$ requires space $\Omega(k \log (n / k))$ to decide which one of the two cases is true for the given $x, y$ pair.

For the lower bound, we use the result of Jayram et al. [19]. Intuitively, the hardest case seems to be to distinguish between exactly $k$ and exactly $k+2$ errors. The main advantage of our lower bound proof is that it formalizes this intuition. Moreover, as our algorithm in Section 3 shows, this bound is tight up to a constant factor for $n \geq k^{2}$ (indeed, for $n \geq k^{1+\epsilon}$ for any $\epsilon>0$ ). This bound is not tight in all situations though, for example when $n \gg k$ but the error is constant. Also, it does not apply to multi-pass algorithms. However, this is better than the earlier bounds [11 by a factor of $\log (n / k)$ for small error.

The rest of the paper is organized as follows: in the next section we give some basic definitions which will be used later in the paper. In Section 3 we give the two randomized one-pass algorithms for testing membership in $\Delta^{\leq k}$ (1-turn-Dyck $\left._{2}\right)$. In 4 we discuss our results regarding testing membership in $\Delta^{\leq k}\left(\right.$ Dyck $\left._{2}\right)$. Our lower bound result is presented in Section 5 .

\section{Definitions and Preliminaries}

\section{$2.1 \ell$-wise independent hash families}

Definition 1. Given positive integers $\ell, n, m$, and $s$, a function $F:\{0,1\}^{s} \rightarrow[m]^{n}$ is an $\ell$-wise independent hash family if given any distinct $i_{1}, i_{2}, \ldots, i_{\ell} \in[n]$ and any (not necessarily distinct) $j_{1}, j_{2}, \ldots, j_{\ell} \in[m]$, we have

$$
\operatorname{Pr}_{r \in\{0,1\}^{s}}\left[F(r)\left(i_{1}\right)=j_{1} \wedge F(r)\left(i_{2}\right)=j_{2} \wedge \cdots \wedge F(r)\left(i_{\ell}\right)=j_{\ell}\right]=\frac{1}{m^{\ell}}
$$

where $F(r)$ is interpreted as a function mapping $[n]$ to $[m]$ in the obvious way.

Lemma 1. [21] For any $\ell, n, m$, there is an $\ell$-wise independent hash family $F:\{0,1\}^{s} \rightarrow[m]^{n}$, with $s=O(\ell \log (n+m))$ with the property that there is a deterministic algorithm which, on input $r \in\{0,1\}^{s}$ and $i \in[n]$, computes $F(r)(i)$ in time poly $(s)$ using space $O(s)$.

\subsection{Some pseudorandom distributions for restricted tests}

Given $m \in \mathbb{N}$, we will denote by $\mathcal{U}_{m}$ the uniform distribution on $\{0,1\}^{m}$.

Definition 2. Given any class $\mathcal{F}$ of boolean functions defined on $\{0,1\}^{m}$, distributions $D_{1}, D_{2}$ over $\{0,1\}^{m}$ and $\delta \in[0,1]$, we say that $D_{1} \delta$-fools $\mathcal{F}$ w.r.t. $D_{2}$ if for all $f \in \mathcal{F}$, we have

$$
\left|\underset{z \sim D_{1}}{\operatorname{Pr}_{1}}[f(z)=1]-\operatorname{Pr}_{z \sim D_{2}}[f(z)=1]\right| \leq \delta
$$


The above concept has been widely studied for many classes of functions, especially in the case when $D_{2}$ is the uniform distribution $\mathcal{U}_{m}$. When $D_{2}$ is the uniform distribution, in many cases, we know of distributions $D_{1}$ with very small support that are nonetheless able to fool some interesting class of functions $\mathcal{F}$ w.r.t. $D_{2}$. We note two such results below, since we will need them later.

Definition 3. Given two vectors $x, y \in\{0,1\}^{m}$, denote by $\langle x, y\rangle$ the $F_{2}$-inner product between $x$ and $y$ : that is, $\langle x, y\rangle=\bigoplus_{i} x_{i} y_{i}$. For $w \in\{0,1\}^{m}$, define the function $L_{w}:\{0,1\}^{m} \rightarrow\{0,1\}$ as follows: $L_{w}(x)=\langle w, x\rangle$. The class of Linear tests on $\{0,1\}^{m}$ is defined to be the class of functions $\left\{L_{w} \mid w \in\{0,1\}^{m}\right\}$.

Lemma 2 (Small bias spaces). [1] Given any $\delta \in \mathbb{R}^{>0}$ and $m \in \mathbb{N}$, there exists an explicit function $G_{1}$ : $\{0,1\}^{s} \rightarrow\{0,1\}^{m}$ for $s=O(\log (m / \delta))$ such that the distribution $G_{1}(r)$ for a randomly chosen $r \in\{0,1\}^{s} \delta$ fools the class of Linear Tests w.r.t. the uniform distribution $\mathcal{U}_{m}$. Moreover, there is a deterministic algorithm $\mathcal{A}$ that, given $r \in\{0,1\}^{s}$ and $i \in[m]$, computes the $i$ th bit of $G_{1}(r)$ in time $\operatorname{poly}(s)$ and space $O(s)$.

The existence of the algorithm $\mathcal{A}$ as stated in Lemma 2 is not formally stated in [1] but easily follows from Construction 3 of such spaces in the paper. We call a $G_{1}$ as described above a $\delta$-biased space over $\{0,1\}^{m}$. The distribution $G_{1}(r)$ for a randomly chosen $r$ is said to be a $\delta$-biased distribution.

The second class of tests we will need to fool is the class of read-once DNF formulae over $\{0,1\}^{m}$. It has been proved recently that $\delta^{\prime}$-biased distributions for small enough $\delta^{\prime}$ can be used to $\delta$-fool the class of read-once DNFs w.r.t. the uniform distribution.

Lemma 3 (Fooling read-once DNFs). [9] Given any $\delta \in \mathbb{R}^{>0}$ and $m \in \mathbb{N}$, any $\delta^{\prime}$-biased distribution $\delta$ fools the class of read-once DNFs over $\{0,1\}^{m}$ w.r.t. the uniform distribution $\mathcal{U}_{m}$, as long as $\delta^{\prime} \leq \frac{1}{m^{\circ(\log (1 / \delta))}}$.

By Lemmas 2 and 3 , we have

Corollary 1. Given any $\delta \in \mathbb{R}^{>0}$ and $m \in \mathbb{N}$, there exists an explicit function $G_{2}:\{0,1\}^{s} \rightarrow\{0,1\}^{m}$ for $s=O(\log m \log (1 / \delta))$ such that the distribution $G_{2}(r)$ for a randomly chosen $r \in\{0,1\}^{s} \delta$-fools the class of Linear Tests w.r.t. the uniform distribution $\mathcal{U}_{m}$. Moreover, there is a deterministic algorithm that, given $r \in\{0,1\}^{s}$ and $i \in[m]$, computes the $i$ th bit of $G_{2}(r)$ in time poly $(s)$ and space $O(s)$.

\section{$3 \quad$ Equivalence with errors}

In this section, we consider the problem of testing membership in $\Delta^{\leq k}\left(1\right.$-turn-Dyck ${ }_{l}$. Magniez et al. [14, showed that it suffices to design efficient streaming algorithms for testing membership in Dyck ${ }_{2}$ in order to get efficient streaming algorithms for testing membership in Dyck . Formally,

Lemma 4 ([14]). If there is a one-pass streaming algorithm for testing membership in Dyck $_{2}$ that uses space $s(n)$ for inputs of length $n$, then there is a one-pass streaming algorithm for testing membership in Dyck $_{l}$ that uses space $O(s(n \log l))$ for inputs of length $n$.

We first prove a lemma similar to Lemma 4, to state that it suffices to design an efficient streaming algorithm for $\Delta \leq k$ (1-turn-Dyck $\left.{ }_{2}\right)\left(\Delta \leq k\right.$ (1-turn-Dyck $\left.\left.{ }_{2}\right)\right)$ in order to get an efficient streaming algorithms for $\Delta^{\leq k}(1$-turn-Dyck $)$ (respectively, $\Delta^{\leq k}\left(\right.$ Dyck $\left.\left._{l}\right)\right)$.

Lemma 5. If there is a one-pass streaming algorithm for testing membership in $\Delta \leq 2 k$ (1-turn-Dyck $\left.{ }_{2}\right)$ $\left(\Delta \leq 2 k\left(\right.\right.$ Dyck $\left.\left._{2}\right)\right)$ that uses space $s(n)$ for inputs of length $n$, then there is a streaming algorithm for testing membership in $\Delta^{\leq k}(1-$ turn-Dyck $)\left(\Delta^{\leq k}\left(\right.\right.$ Dyck $\left.\left._{l}\right)\right)$ that uses space $O(s(n l))$ for inputs of length $n$.

Proof. We use a distance preserving code for this. We encode an opening parenthesis of type $\left({ }_{i}\right.$ by $\left({ }^{i-1}\left[\left(^{l-i}\right.\right.\right.$. And we encode a closing parenthesis of type $)_{i}$ by $\left.\left.)^{l-i}\right]\right)^{i-1}$. Now given a string $w \in \Sigma_{l}^{n}$, the new string $w^{\prime}$ is over the alphabet $\Sigma=\{(,[)]$,$\} . And \left|w^{\prime}\right|=n l$. Also for every mis-match in $w, w^{\prime}$ has two mis-matches. Thus the lemma. 
Let $D^{\leq k}\left(1\right.$-turn-Dyck $\left._{2}\right)$ be the set of string obtained by changing at most $k$ symbols of words in

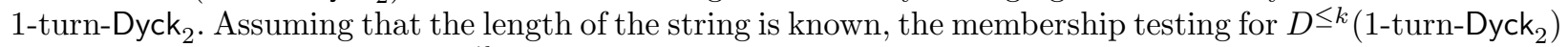
(which is more general than $\Delta^{\leq k}\left(1\right.$-turn-Dyck $\left.k_{2}\right)$ can also be handled by the techniques introduced in the paper. If the input string has opening parenthesis in the first half of the string, then it is considered to be an error. It is easy to keep track of such errors.

We now note that $\Delta^{\leq k}\left(1\right.$-turn-Dyck ${ }_{2}$ ) on inputs of length $n$ reduces to the problem $\operatorname{Ham}_{n / 2, k}$.

Lemma 6. There is a deterministic one-pass streaming algorithm that uses space $O(\log n)$ and time $O(n)$, which given as input a string $w \in\{(,[,),]\}^{n}$, outputs a pair of strings $x, y \in\{0,1\}^{n / 2}$ and either accepts or rejects. If the algorithm rejects, we have $w \notin \Delta^{\leq k}\left(1\right.$-turn-Dyck $\left.{ }_{2}\right)$. Otherwise, we have $\Delta\left(x, y^{R}\right) \leq k$ iff $w \in \Delta^{\leq k}\left(1-\right.$ turn -Dyck $\left.{ }_{2}\right)$.

Proof. Given an input of length $n$, the algorithm scans its input from left to right, and outputs 0 on seeing "(" and 1 on seeing "[" in the first $n / 2$ symbols of its input $w$; similarly, on the second half of $w$, the algorithm outputs 0 on seeing ")" and 1 on seeing "]". The algorithm rejects either if it sees the closing braces in the first half of its input or the opening braces in the second half of its input (in this case, an opening brace has been corrupted by a closing brace or vice versa) and accepts otherwise. If the algorithm accepts, we see that $\Delta\left(x, y^{R}\right)$ is exactly the distance of the input from a string in 1-turn-Dyck $\mathbf{k}_{2}$. The lemma follows.

The above lemma shows that it suffices to come up with a streaming algorithm for the Hamming distance problem to solve the problem $\Delta^{\leq k}$ (1-turn-Dyck ${ }_{2}$ ). Once we have such an algorithm, we simply run the above reduction on an input $w \in\{(,[,),]\}^{n}$, and obtain strings $x, y^{R}$, which we feed in as input to the algorithm for $\mathrm{Ham}_{n / 2, k}$ (of course, if the reduction rejects, we reject the input). Though $\mathrm{Ham}_{n, k}$ is only a minor restatement of $\Delta^{\leq k}$ (1-turn-Dyck ${ }_{2}$ ), we prefer to work with this problem because of its cleaner definition.

Theorem 2. For any $k$ and any constant $c>0$, there is a one-pass randomized streaming algorithm which, when given as input strings $\left(x, y^{R}\right) \in\{0,1\}^{n} \times\{0,1\}^{n}$, that accepts with probability 1 if $\Delta(x, y) \leq k$ and rejects with probability $1-1 / n^{c}$ if $\Delta(x, y)>k$. The algorithm also detects the locations where $x$ and $y$ differ with probability at least $1-1 / n^{c}$ if $\Delta(x, y) \leq k$. The algorithm uses $O(k \log n)$ space and $O(k \log n)$ randomness. The time required by the algorithm is poly $(k \log n)$ per item plus $n^{k+O(1)}$ for post-processing.

Proof. The algorithm uses a fingerprinting strategy and is directly inspired by the standard randomized communication complexity protocol for the Equality problem (see [13], for example). Fix a field $\mathbb{F}_{2^{\ell}}$, where the exact value of $\ell$ will be determined later. We call a polynomial $p(z) \in \mathbb{F}_{2^{\ell}}[z]$ boolean if all of its coefficients are 0 or 1 . The weight of a boolean polynomial $p$ will be the number of non-zero coefficients of $p$.

We think of $w \in\{0,1\}^{n}$ as defining a boolean polynomial $p_{w}(z) \in \mathbb{F}_{2^{\ell}}[z]$ as follows: $p_{w}(z)=\sum_{i=1}^{n} w_{i} z^{i-1}$, where $w_{i}$ denotes the $i$ th bit of $w$. Note that the polynomial $q_{x, y}(z):=p_{x}(z)+p_{y}(z)$ is a boolean polynomial of weight exactly $\Delta(x, y)$. We check that $\Delta(x, y) \leq k$ by evaluating $q_{x, y}(z)$ at a random $\alpha \in \mathbb{F}_{2^{\ell}}$. More formally, the algorithm is:

- Pick $\alpha \in \mathbb{F}_{2^{e}}$ uniformly at random.

- Check if $q_{x, y}(\alpha)=p(\alpha)$ for any boolean polynomial $p$ of degree less than $n$ and weight at most $k$. If not, REJECT.

- If the above does hold for some boolean polynomial of weight at most $k$, ACCEPT and pick any such polynomial $p(z)=\sum_{i} p_{i} z^{i}$. Let $S=\left\{i \quad p_{i} \neq 0\right\}$ be the support of $p$. Output $S$ as the estimate of points where $x$ and $y$ differ.

Let us first establish the correctness of the above algorithm (assuming $\ell$ is large enough). Clearly, if $\Delta(x, y) \leq k$, then $q_{x, y}(z)$ is a polynomial of weight at most $k$ and the algorithm always accepts. The algorithm can only err if: (a) $\Delta(x, y)>k$ or (b) $\Delta(x, y) \leq k$ but the algorithm outputs the wrong set of indices as its estimate of where $x$ and $y$ differ. In either case, there is a boolean polynomial $p(z)$ of degree less than $n$ and weight at most $k$ such that $q_{x, y}(z) \neq p(z)$ but $q_{x, y}(\alpha)=p(\alpha)$. For any fixed polynomial $p(z)$, this happens with probability at most $n / 2^{\ell}$ by the Schwartz-Zippel Lemma. Since the number of polynomials 
of weight at most $k$ is at most $n^{k}$, the probability that there exists any such polynomial $p$ is bounded by $n^{k+1} / 2^{\ell}$. Choosing $\ell=O(k \log n)$, we can reduce this error to $1 / n^{c}$ as claimed.

Computing $q_{x, y}(\alpha)$ can easily be done in a one-pass fashion using space $O(\ell)=O(k \log n)$ and time poly $(k \log n)$ per item. After reading the stream, we need to cycle through the $n^{k}$ boolean polynomials $p$ of weight at most $k$ and compute the values they take at input $\alpha \in \mathbb{F}_{2^{\ell}}$, which can also be done in space $O(k \log n+\ell)=O(k \log n)$ and time $n^{k} \operatorname{poly}(k \log n)=n^{k+O(1)}$, as claimed above. This completes the proof of the theorem.

\subsection{A randomness-efficient streaming algorithm for Hamming Distance}

Above, we showed that $\operatorname{Ham}_{n, k}$ can be computed using space $O(k \log n)$ and $O(k \log n)$ random bits. Are these parameters optimal? As we will show later in Section 5 , the bound on space is nearly optimal. However, we show in this section that the number of random bits can be significantly reduced, if one is willing to use a small amount of additional space. The ideas in this section go back to the results of Yao [22] and Huang et al. [1], who designed efficient randomized communication complexity protocols for the two-party problem of checking if the Hamming Distance between $x$ and $y$ is at most $k$.

Let $\mathrm{PHam}_{n, k, l}:\{0,1\}^{n} \times\{0,1\}^{n} \rightarrow\{0,1\}$ be a partial function, defined as follows: On input $\left(x, y^{R}\right)$ it evaluates to 0 if the hamming distance between $x$ and $y$ is greater than or equal to $l$, it evaluates to 1 if the distance is less than or equal to $k$ and is not defined on other inputs.

Theorem 3. For every constant $0 \leq \epsilon \leq 1$ there is a randomized one-pass streaming algorithm that computes $\mathrm{Ham}_{n, k}$ using $O\left(k^{1+\epsilon}+\log n\right)$ space and $O(\log n)$ randomness and errs with probability bounded by $1 / 8$. The time taken by the algorithm is $\left.O(\log n)^{O(1)}+k^{O(1)}\right)$ per item.

Proof Strategy: In order to prove the above theorem, we divide the problem into two parts. Assuming there are at most $2 k$ errors, we design an algorithm that computes Ham $_{n, k}$ correctly with high probability. We call this the inner algorithm. We design another randomized algorithm to compute $\mathrm{PHam}_{n, k, 2 k}$ with high probability. We call this algorithm the outer algorithm.

We output 1 , that is we declare that the number of indices where $x$ and $y$ differ is at most $k$, if and only if both the inner and the outer algorithms output 1 . If $x$ and $y$ differ on more than $2 k$ indices, then the outer algorithm will output 0 with high probability. The answer of the inner algorithm will not be reliable in this case. Where as if they differ on more than $k$ but less than $2 k$ places then the inner algorithm will output 0 with high probability. Let $\gamma_{1}, \gamma_{2}$ be errors in inner and outer algorithms respectively. Then the overall error $\gamma$ is bounded by $\gamma_{1}+\gamma_{2}$. We prove that both $\gamma_{1}$ and $\gamma_{2}$ are bounded by $\gamma / 2$ for a small constant $\gamma$.

\section{Inner algorithm}

Definition 4. Given, $k, n \in \mathbb{N}$, we say that an element $w \in([k] \times\{0,1\})^{n}$ is an XOR representation of length $n$ of a string $a \in\{0,1\}^{k}$ if for each $j \in[k]$, we have $a_{j}=\bigoplus_{i: w_{i}=\left(j, u_{i}\right)} u_{i}$.

We think of the XOR representation as streaming updates of a structure over $\mathbb{F}_{2}$.

Lemma 7. There is a randomized one-pass streaming algorithm which given input $x, y^{R} \in\{0,1\}^{n}$ such that $\Delta(x, y) \leq 2 k$ computes an XOR representation of length $n$ of $a, b \in\{0,1\}^{16 k^{2} / \gamma}$ such that with probability $1-\gamma / 4, \operatorname{Ham}_{n, k}(x, y)=\operatorname{Ham}_{16 k^{2} / \gamma, k}(a, b)$ The algorithm uses $O(\log n)$ bits of randomness, $O(\log n)$ space, and $(\log (n / \gamma))^{O(1)}$ time per item.

Proof. The proof is simple. We pick a random hash function $h$ from a pairwise independent hash family of functions mapping $[n]$ to $\left[16 k^{2} / \gamma\right]$. We think of $h$ as dividing the $n$ indices of $x$ and $y$ into $16 k^{2} / \gamma$ buckets.

Given $x, y$ such that $\Delta(x, y) \leq 2 k$, call index $i \operatorname{good}$ if $x_{i} \neq y_{i}$. Given two good indices $i \neq j$, the probability that $h$ maps both of them to the same bucket is at most $\gamma / 16 k^{2}$. A simple union bound tells us that with probability $1-\gamma / 4$, all the good indices are mapped to different buckets. 
After having picked $h$, the streaming algorithm computes the XOR representations of $a, b$ defined as follows: for any $j, a_{j}$ is the XOR of the bits of $x$ whose indices are in the $j$ th bucket; formally, $a_{j}=$ $\bigoplus_{i: h(i)=j} x_{i}$; the string $b$ is similarly related to $y$. Clearly, if $h$ maps the good indices to different buckets, then $a_{j} \neq b_{j}$ iff the $j$ th bucket contains a good index and hence $\Delta(a, b)=\Delta(x, y)$. On reading the input bit $x_{i}$, the algorithm computes the bucket $j=h(i)$ and writes down $\left(j, x_{i}\right)$ which in effect updates the $j$ th bit of $a$. In a similar way, when scanning $y$, the algorithm updates $b$.

The space and randomness requirements are easily analyzed. Picking a random hash function $h$ from a pairwise independent family as above requires $O\left(\max \left\{\log n, \log \left(k^{2} / \gamma\right)\right\}\right)=O(\log (n / \gamma))$ random bits by Lemma 1. The algorithm needs to store these random bits only. Computing $h(j)$ for any $j$ only requires space $O(\log n / \gamma)$. Finally, the processing time per element is $O(\operatorname{poly}(\log (n / \gamma))$.

We will use the above algorithm as a streaming reduction and solve the problem using the algorithm of Lemma 8 .

Lemma 8. For any $n, k$ and every constant $0<\epsilon<1$ and $\gamma \geq \frac{1}{k^{O(1)}}$, there is a randomized one-pass streaming algorithm which, on inputs $a, b \in\{0,1\}^{16 k^{2} / \gamma}$ accepts iff $\Delta(a, b) \leq k$ with error probability at most $\frac{\gamma}{4}$. The algorithm uses $O(\log k)$ bits of randomness, $O\left(k^{1+\epsilon}+\log n\right)$ space, and time per element is $k^{O(1)}$. The algorithm expects its inputs $a, b$ to be given in terms of XOR representations of length $n$.

Proof. First, we present the algorithm for the special case when $n=k$ and the input is simply the pair of strings $a, b$ in the natural order of increasing indices. We will then explain the simple modifications that are necessary for the case when the input is a pair of XOR representations of length $n$.

Fix a positive constant $\delta<\epsilon$. Let $h:\left[16 k^{2} / \gamma\right] \rightarrow\left[k^{1+\delta}\right]$ be a function picked at random. Let $j \in\left[k^{1+\delta}\right]$ be a fixed bucket. We have $\operatorname{Pr}[h(i)=j]=\frac{1}{k^{1+\delta}}$.

Define a set $I$ of indices as follows: if $\Delta(a, b) \leq k$, then let $I$ be the indices where $a$ and $b$ differ; otherwise, let $I$ be any set of $k+1$ indices where $a$ and $b$ differ. Let $u$ be the size of a subset $U$ of $I$. We have $\operatorname{Pr}[h(U)=j] \leq \frac{1}{\left(k^{1+\delta}\right)^{u}}$. By a union bound over $U$ of size $u, \operatorname{Pr}[\exists U: h(U)=j] \leq \frac{\left(\begin{array}{c}k+1 \\ \left(k^{1+\delta}\right)\end{array}\right.}{{ }^{u}} \leq \frac{(k+1)^{u}}{\left(k^{1+\delta}\right)^{u}} \leq \frac{1}{k^{\delta u / 2}}$. Therefore, since there are at most $k^{2}$ buckets, $\operatorname{Pr}[\exists U \exists$ a bucket $j: h(U)=j] \leq 1 / k^{\delta u / 2-2}$.

We want this probability to be less than $\gamma / 8$. Therefore we select $u=O\left(1 / \delta+\frac{\log (1 / \gamma)}{\log k}\right)=O(1)$, where the last equality uses $\gamma \geq \frac{1}{k^{O(1)}}$ and $\delta$ is a constant.

Note that the above argument works if we used a function from a $u$-wise independent family of functions rather than a random function. This requires only $O(u \log (k / \gamma))=O(\log k)$ bits of randomness and space $O(\log (k / \gamma))$ by Lemma 1. Hereafter, we assume that we have picked a hash function $h$ from this family so that each bucket $j \in\left[k^{1+\delta}\right]$ contains at most $u$ indices from $I$. Let $B_{j}^{a}$ and $B_{j}^{b}$ be the buckets formed by hashing $a$ and $b$ respectively, where $1 \leq j \leq k^{1+\delta}$.

Given boolean strings $a^{\prime}, b^{\prime}$ of the same length, define $\Delta_{u}\left(a^{\prime}, b^{\prime}\right)$ to be $\min \left\{\Delta\left(a^{\prime}, b^{\prime}\right), u\right\}$. We will compute the function $F(a, b)=\sum_{j \in\left[k^{1+\delta}\right]} \Delta_{u}\left(B_{j}^{a}, B_{j}^{b}\right)$ and accept if the value computed is at most $k$. It can easily be seen, using the properties of $h$, that this computes $\operatorname{Ham}_{16 k^{2} / \gamma, k}(a, b)$.

Computing $\Delta_{u}\left(B_{j}^{a}, B_{j}^{b}\right)$ for any $j$ is easily done using the ideas of the algorithm of Theorem 2. We work over the field $\mathbb{F}_{2^{\ell}}$ where $\ell$ is a parameter that we will fix shortly. Given a polynomial $p \in \mathbb{F}_{2^{\ell}}[z]$ with only 0-1 coefficients, we denote by the weight of $p$ the number of non-zero coefficients of $p$. Given $c \in\{a, b\}$ and $j \in\left[k^{1+\delta}\right]$, the bucket $B_{j}^{c}$ defines for us the polynomial $p_{j, c}(z)=\sum_{i \in h^{-1}(j)} c_{i} z^{i}$ over $\mathbb{F}_{2^{\ell}}$. Define the polynomial $q_{j}(z)=p_{j, a}(z)+p_{j, b}(z)$. The weight of $q_{j}$ is exactly $\Delta\left(B_{j}^{a}, B_{j}^{b}\right)$. The algorithm to compute $F(a, b)$ is the following:

1. Pick $\alpha \in \mathbb{F}_{2^{\ell}}$ uniformly at random.

2. For each $j$, compute $q_{j}(\alpha)$ and check if it evaluates to the same value as some polynomial $p$ of weight at most $u$. If so, let $w_{j}$ be the weight of an arbitrary such $p$; if not, let $w_{j}=u$.

3. Output $\sum_{j} w_{j}$.

The above algorithm errs on bucket $j$ only if there is a polynomial $p \neq q_{j}$ of weight at most $u$ such that $p(\alpha)=q_{j}(\alpha)$. This occurs with probability at most $O\left(\frac{k^{2}}{\gamma 2^{\ell}}\right)$ for a fixed polynomial $p$ and hence with 
probability at most $\frac{(k / \gamma)^{O(u)}}{2^{\ell}}$ for some polynomial $p$ of weight at most $u$ after a union bound. After taking a union bound over buckets, we get a failure probability of at most $\frac{(k / \gamma)^{O(u)}}{2^{\ell}}$ (with different constants in the exponents). Choosing $\ell=O\left(u \log \left(\frac{k}{\gamma}\right)\right)=O(\log k)$, we can reduce the error to $\gamma / 8$.

The overall error of the algorithm is at most $\gamma / 4$. The space used per bucket and the number of random bits used is at most $O(\log k)$. Adding up over all buckets, the space used is bounded by $O\left(k^{1+\delta} \log k\right)$, which is at most $O\left(k^{1+\epsilon}\right)$. The time taken by the algorithm to compute the values $\left\{q_{j}(\alpha) \mid j \in\left[k^{1+\delta}\right]\right\}$ is $k^{O(1)}$. Finally, checking if each $q_{j}(\alpha)$ evaluates to the same value as a polynomial of weight at most $u$ takes time $k^{O(u)}=k^{O(1)}$.

Now for the case when the input is given as a pair of XOR representations of length $n$. We simply note that the polynomials $p_{j, a}$ and $p_{j, b}$ are still easy to compute. For example, on reading the $i$ th element $w_{i}=\left(j_{i}, u_{i}\right)$ of the XOR representation of $a$, the algorithm simply updates the current value of $p_{h\left(j_{i}\right), a}(\alpha)$ by adding $u_{i} \alpha^{j_{i}}$ to it; this works as intended since $\mathbb{F}_{2^{\ell}}$ is a field of characteristic 2 . The algorithm only needs an additional counter that counts up to $n$ so that it knows when the XOR representation of $a$ ends.

Setting $\gamma$ to be $1 / 8$ in the Lemmas 7 and 8 , we see that the space taken by the Inner algorithm overall is $O\left(k^{1+\epsilon}+\log n\right)$, the amount of randomness used is $O(\log n)$ and the time taken per item is $O\left((\log n)^{O(1)}+\right.$ $\left.k^{O(1)}\right)$.

Outer algorithm Given $x, y \in\{0,1\}^{n}$, we denote by $\langle x, y\rangle$ the $\mathbb{F}_{2}$-inner product of $x$ and $y$. Formally, $\langle x, y\rangle=\bigoplus_{i=1}^{n} x_{i} y_{i}$.

Lemma 9. There is a randomized one-pass streaming algorithm that computes $\mathrm{PHam}_{n, k, 2 k}$ correctly with probability $1-\gamma / 2$ using $O(\log n \log (1 / \gamma))$ bits of space and randomness and time per item $(\log n)^{O(1)} \log (1 / \gamma)$.

Proof. For simplicity, we will assume that $k$ is a power of 2 . All the results carry through in the general case, with only superficial changes.

We use a protocol of Yao 22. Yao devised a one-way 5 randomized communication complexity protocol using which two players Alice and Bob, given inputs $x$ and $y$ respectively, can decide $\operatorname{PHam}_{n, k, 2 k}(x, y)$ using $O(\log (1 / \gamma))$ bits of communication. A brief sketch follows. Let $u$ denote $x \oplus y$. Using public randomness, Alice and Bob pick random strings $z_{1}, \ldots, z_{\ell} \in\{0,1\}^{n}$ for $\ell=O(\log (1 / \gamma))$ such that each bit of each $z_{i}$ is set to 1 independently with probability $1 / 4 k$ - we call this distribution $D_{1 / 4 k}$. Alice computes $\left\langle x, z_{1}\right\rangle, \ldots,\left\langle x, z_{\ell}\right\rangle$ and sends them to Bob who uses them to compute $\left\langle u, z_{1}\right\rangle, \ldots,\left\langle u, z_{\ell}\right\rangle$.

Let $z_{i}$ be picked from $D_{1 / 4 k}$. It is easily checked that if $\Delta(x, y) \leq k$, then $\left\langle u, z_{i}\right\rangle$ takes value 1 with probability at most $p_{1}=\frac{\left(1-1 / \sqrt{e}+o_{k}(1)\right)}{2}$. On the other hand, if $\Delta(x, y) \geq 2 k$, then $\left\langle u, z_{i}\right\rangle$ takes value 1 with probability at least $p_{2}=\frac{\left(1-1 / e+o_{k}(1)\right)}{2}$. Thus, by performing a suitable threshold on the number of $i$ such that $\left\langle u, z_{i}\right\rangle=1$ - say by checking if the number of $1 \mathrm{~s}$ is at least $\frac{p_{1}+p_{2}}{2}-$ Bob can compute $\mathrm{PHam}_{n, k, 2 k}(x, y)$ correctly with error probability at most $\frac{\gamma}{2}$.

Using the above ideas, we wish to come up with a streaming algorithm for this problem that is also randomness efficient. Both these constraints require us to change the original protocol. (Note that the obvious implementation of the above protocol in the streaming setting will require $\Omega(n \ell)$ bits of space and randomness.) To reduce the amount of randomness, we run Yao's protocol with pseudorandom $z$ from a distribution $D$ that fools the linear test defined by the string $u$ (see Section 2 for the definition of a "linear test"). Formally, we want a distribution $D$ over $\{0,1\}^{n}$ such that for any $w \in\{0,1\}^{n}$

$$
\left|\operatorname{Pr}_{z \sim D}[\langle w, z\rangle=1]-\operatorname{Pr}_{z \sim D_{1 / 4 k}}[\langle w, z\rangle=1]\right| \leq 1 / 100
$$

That is, we want a distribution $D$ that $1 / 100$-fools linear tests w.r.t. $D_{1 / 4 k}$. Furthermore, we would like to be able to sample from $D$ using a small number of random bits.

\footnotetext{
${ }^{5}$ Actually, Yao's protocol works in the more restrictive simultaneous message model, but this fact is not relevant here.
} 
Fix $w \in\{0,1\}^{n}$. Let $f_{w}$ be the following related test, defined on $\{0,1\}^{n t}$ :

$$
f_{w}\left(z_{11}^{\prime}, z_{12}^{\prime}, \ldots, z_{1 t}^{\prime}, z_{21}^{\prime}, z_{22}^{\prime}, \ldots, z_{2 t}^{\prime}, \ldots, z_{n 1}^{\prime}, z_{n 2}^{\prime}, \ldots, z_{n t}^{\prime}\right)=\bigoplus_{i: w_{i}=1}\left(\bigwedge_{j=1}^{l} z_{i j}^{\prime}\right)
$$

where $2^{t}=4 k$. Note that $\operatorname{Pr}_{z \in D_{1 / 4 k}}[\langle w, z\rangle=1]=\operatorname{Pr}_{z^{\prime} \in \mathcal{U}_{n t}}\left[f_{w}\left(z^{\prime}\right)=1\right]$, where $\mathcal{U}_{r}$ denotes the uniform distribution on $\{0,1\}^{r}$.

We will first design a distribution $D^{\prime}$ over $\{0,1\}^{n t}$ that $1 / 100$-fools the family of tests $\left\{f_{w} \mid w \in\{0,1\}^{n}\right\}$ w.r.t. the uniform distribution $\mathcal{U}_{n t}$.

Now, we describe $D^{\prime}$. We break the variables $\left\{z_{i j}^{\prime}\right\}$ into two blocks: $B_{1}:=\left\{z_{i 1}^{\prime} \mid i \in[n]\right\}$ and $B_{2}:=\left\{z_{i j}^{\prime} \mid i \in\right.$ $[n], j \neq 1\}$. Consider the test $f_{w}$ evaluated at a random input $z^{\prime} \in\{0,1\}^{n t}$. It is helpful to view this evaluation as a two-step process: In the first step, we substitute a uniform random string $z_{2}^{\prime}$ for the tuple of variables in $B_{2}$. After this substitution, $f_{w}\left(\cdot, z_{2}^{\prime}\right)$ becomes a linear function on the variables in $B_{1}$. If this linear function is the zero linear function, then $f_{w}\left(z_{1}^{\prime}, z_{2}^{\prime}\right)$ cannot evaluate to 1 on any setting $z_{1}^{\prime}$ of the variables in $B_{1}$. On the other hand, if this linear function is non-zero, then $f_{w}\left(z_{1}^{\prime}, z_{2}^{\prime}\right)$ evaluates to 1 with probability exactly $1 / 2$ over the choice of $z_{1}^{\prime}$. Putting things together, we see that $\operatorname{Pr}_{z^{\prime}}\left[f_{w}\left(z^{\prime}\right)=1\right]=(1 / 2) \operatorname{Pr}_{z_{2}^{\prime}}\left[g_{w}\left(z_{2}^{\prime}\right)=1\right]$, where $g_{w}$ is the following read-once DNF formula on the variables in $B_{2}$ that tells us exactly when $f_{w}$ becomes a non-zero linear function on the variables in $B_{1}: g_{w}=\bigvee_{i: w_{i}=1} \bigwedge_{j=2}^{l} z_{i j}^{\prime}$

This tells us that we only need to fool read-once DNFs and linear tests w.r.t. the uniform distribution to fool $f_{w}$-tests w.r.t. the uniform distribution. We will generate $z^{\prime} \in D^{\prime}$ as follows: $z_{1}^{\prime}$ will be sampled from an explicit $\delta_{1}$-biased space $D_{1}^{\prime}$ and $z_{2}^{\prime}$ will be independently sampled from an explicit space $D_{2}^{\prime}$ that $\delta_{2}$-fools read once DNFs. We have:

$$
\begin{aligned}
& \left|\operatorname{Pr}_{z^{\prime} \sim D^{\prime}}\left[f_{w}\left(z^{\prime}\right)=1\right]-\operatorname{Pr}_{z^{\prime} \sim \mathcal{U}_{n t}}\left[f_{w}\left(z^{\prime}\right)=1\right]\right| \\
& =\left|\underset{z_{2}^{\prime} \sim D_{2}^{\prime}}{\operatorname{Pr}}\left[g_{w}\left(z_{2}^{\prime}\right)=1\right] \cdot \operatorname{Pr}_{z_{1}^{\prime} \sim D_{1}^{\prime}}\left[f_{w}\left(z_{1}^{\prime}, z_{2}^{\prime}\right)=1 \mid g_{w}\left(z_{2}^{\prime}\right)=1\right]-\frac{\operatorname{Pr}_{z_{2}^{\prime} \sim \mathcal{U}_{n(t-1)}}\left[g_{w}\left(z_{2}^{\prime}\right)=1\right]}{2}\right| \\
& \leq\left|\operatorname{Pr}_{z_{2}^{\prime} \sim D_{2}^{\prime}}^{\operatorname{Pr}}\left[g_{w}\left(z_{2}^{\prime}\right)=1\right]-\underset{z_{2}^{\prime} \sim \mathcal{U}_{n(t-1)}}{\operatorname{Pr}}\left[g_{w}\left(z_{2}^{\prime}\right)=1\right]\right|+\left|\operatorname{Pr}_{z_{1}^{\prime} \sim D_{1}^{\prime}}\left[f_{w}\left(z_{1}^{\prime}, z_{2}^{\prime}\right)=1 \mid g_{w}\left(z_{2}^{\prime}\right)=1\right]-\frac{1}{2}\right| \\
& \leq \delta_{1}+\delta_{2}
\end{aligned}
$$

where the first inequality uses the fact that $|p q-r s| \leq|p-r|+|q-s|$ for any $p, q, r, s \in[0,1]$, and the second inequality follows from the definitions of $D_{1}^{\prime}$ and $D_{2}^{\prime}$. Choosing $\delta_{1}$ and $\delta_{2}$ to be small enough constants, we obtain a pseudorandom distribution $D^{\prime}$ that $1 / 100$-fools $f_{w}$-tests w.r.t. the uniform distribution. By Lemma 2 and Corollary 1, the amount of randomness required for the above is $O(\log n)$. Using $D^{\prime}$, we can define a distribution $D$ that fools linear tests w.r.t. $D_{1 / 4 k}$ as follows: to pick $z \sim D$, we pick $z^{\prime} \sim D^{\prime}$ and output $z$ defined by $z_{i}=\wedge_{j=1}^{t} z_{i j}^{\prime}$ for each $i$. It is easily seen from the definition of the tests $f_{w}$ that the distribution $D$ $1 / 100$-fools all linear tests w.r.t. $D_{1 / 4 k}$. Since no additional randomness is used to generate $D$, the amount of randomness used is $O(\log n)$. Moreover, by Lemma 2 and Corollary 1 given a random seed $r$ of length $O(\log n)$ and $j \in[n]$, the $j$ th bit of the output of $D$ on this random seed can be generated using $O(\log n)$ bits of space in time poly $(\log n)$.

With the pseudorandom distribution $D$ in place, we can run Yao's protocol in the streaming setting with independent random strings $z_{1}, \ldots, z_{\ell}$ picked from the distribution $D$. Exactly as above, for suitably chosen $\ell=O(\log (1 / \gamma))$, this algorithm computes $\operatorname{PHam}_{n, k, 2 k}(x, y)$ with error probability at most $\gamma / 2$. The space and randomness used are both $O(\log n \log 1 / \gamma)$ and the time taken is $n$ poly $(\log n) \log (1 / \gamma)$. Setting $\gamma=1 / 8$, this proves the lemma and also concludes the proof of Theorem 3.

\section{Accepting Dyck ${ }_{2}$ with errors}

In this section we consider the membership problem of $\Delta^{\leq k}\left(\right.$ Dyck $\left._{2}\right)$. We assume that disregarding the type of the brackets the string is well-matched. We only consider the kind of errors where an opening(closing) 
parenthesis of one type is replaced by an opening(closing, respectively) parenthesis of the other type. We prove the following theorem:

Theorem 4. For any $k$ there exists a constant $c>0$, there is a randomized one-pass algorithm such that, given a string $w \in \Sigma^{n}$, if $w \in \Delta^{\leq k}\left(\right.$ Dyck $\left._{2}\right)$ then with probability at least $1-1 / n^{c}$ it accepts $w$ and if $w \notin \Delta^{\leq k}\left(\right.$ Dyck $\left._{2}\right)$, then with probability $1-1 / n^{c}$ it rejects $w$. The algorithm uses $O(k \log n+\sqrt{n \log n})$ space and takes poly $(k \log n)$ time per item and $n^{k+O(1)}$ time for post-processing.

It is easy to see that combining the ideas from [14] and from the previous section, we can accept $\Delta^{\leq k}\left(\right.$ Dyck $\left._{2}\right)$ using $O(k \sqrt{n \log n})$ space. But for the bound stated in Theorem 4 , we need more work.

In [14] a one-pass randomized streaming algorithm was given for the membership testing of Dyck ${ }_{2}$. We refer to that as the MMN algorithm. We make one change to the MMN algorithm. We use the stack only to store indices from the set $[n]$, and do not store the partial evaluations of a polynomial on the stack.

Divide the input into $\sqrt{n / \log n}$ blocks of length $\sqrt{n \log n}$ each. In each block, check for balanced parentheses and if there are less than or equal to $k-E r r$ mis-matches, then reduce the remaining string to a string (possibly empty) of closing parentheses followed by a string (possibly empty) of opening parentheses. Here, Err is a counter that maintains the number of mismatches found so far. If Err exceeds $k$, then halt the algorithm and reject.

Let $x$ denote the reduced string obtained by matching parentheses within each block. (Note that this can be done in a streaming fashion.) For the reduced string $x$ we say that the opening parenthesis at position $i$ has an index $h_{x, i}$ if it is the $h$ th opening parenthesis in $x$. We say that the closing parenthesis has index $h_{x, i}$ if it is the the closing parenthesis that closes an opening parenthesis having index $h_{x, i}$ in $x$. We drop $x$ when it is clear from the context.

Observation 1 Note that no two opening (or two closing) parentheses have the same index.

Also, an opening parenthesis has the same index as another closing parenthesis if and only if they form a matching pair in the string obtained from the input string by disregarding the type of the parentheses.

For example in the input (([]) []) the indices of the opening parentheses would be $\left({ }^{1}\left({ }^{2}\left[{ }^{3}\right]\right)\left[{ }^{4}\right]\right)$ and the indices of the opening and closing parentheses would be $\left({ }^{1}\left({ }^{2}\left[{ }^{3}\right]^{3}\right)^{2}\left[{ }^{4}\right]^{4}\right)^{1}$. If we reorder the input such that all opening parentheses are in the first half with ascending index and the closing parentheses are in the second half with descending input we get $\left({ }^{1}\left({ }^{2}\left[{ }^{3}[]^{4}\right]^{4}\right)^{2}\right)^{1}$.

We now describe the procedure for computing the index. The index of an opening parenthesis is easy to compute. It is a monotonically increasing quantity and can be stored in a $O(\log n)$ bit counter say $c_{o p e n}$. It is initialized to 0 and incremented by 1 every time an opening parenthesis is encountered. To compute the index of a closing parenthesis we use the stack. The stack is only being used to compute the index. At any stage during the algorithm we maintain the intervals of yet to be matched open parentheses on the stack.

The first block consists of only opening parentheses (assuming we have already reduced the block). After processing the first block we push $\left[1, c_{\text {open }}\right]$ on the stack to remember the interval of indices yet to be matched. (In the case that all parentheses of the first block are matched within the first block, the next block is treated as the first block).

Now suppose we process the next block (also assume that this block is already reduced). A reduced block will consist of a sequence of closing parentheses (possible empty) followed by a sequence of opening parentheses (possible empty). Recall we maintain the intervals yet to be matched on the stack, say the stack-top is $\left[m, m^{\prime}\right]$.

If the block begins with a (non-empty) string of closing parentheses, $m^{\prime}$ is the index of the first closing parenthesis, and is decremented after reading the closing parenthesis. As long as $m^{\prime} \geq m$, this continues. If $m^{\prime}<m$ we get the next interval of unmatched parenthesis from the stack. (If the stack is empty the input is not well matched disregarding the type of parentheses and we reject the input). If the string of closing parentheses ends while $m \geq m^{\prime}$, we push the remaining interval to the stack.

When reading the string of opening parentheses we let $m=c_{\text {open }}$ at the beginning, process all opening parentheses, and before moving on to the next block we push $\left[m, c_{\text {open }}\right]$ (where $c_{\text {open }}$ is updated value after reading the string of opening parentheses). 
While processing closing parentheses, the stack is, if at all, popped but never pushed. While processing opening parentheses, a new stack element may be pushed. However, this happens at most once per block, and therefore at most $\sqrt{n / \log n}$ times. Also the elements of the stack are tuples of indices so they are $O(\log n)$ bits. Therefore, the total space needed to compute indices is $O(\log n \sqrt{n / \log n})=O(\sqrt{n \log n})$.

So we can compute the index of the parentheses, and now we show how to use this to compute $\Delta^{\leq k}\left(\right.$ Dyck $\left._{2}\right)$. Now assume that, at any stage $i$, we have the index of the input symbol $x_{i}$. Let the sign of the opening parentheses be +1 and that of closing parentheses be -1 . We think of the reduced string $x \in\{(,[,),]\}^{*}$ as a string over $\{0,1\}^{*}$ by replacing every occurrence of '(' and ')' by a 0 and every occurrence of '[' and ']' by a 1. We think of this string defining a Boolean polynomial $p_{x}(z)=\sum_{i} \operatorname{sign}\left(x_{i}\right) x_{i} z^{\text {index of } x_{i}}$. Due to Observation 1. it is easy to see that the weight of the polynomial $p_{x}$ is at most $k-E r r$ if and only if $w \in \Delta \leq k\left(\right.$ Dyck $\left._{2}\right)$. We now check whether $w \in \Delta^{\leq k}\left(\right.$ Dyck $\left._{2}\right)$ by evaluating $p_{x}$ at a random $\alpha \in \mathbb{F}_{2^{l}}$. Assuming that we know how to compute index of $x_{i}$ at step $i$, we can evaluate $p_{x}$ as in the proof of Theorem 2

Given below is the algorithm that uses the index finding procedure as described above, and evaluates polynomial $p_{x}$ at a random location to test membership of $w$ in $\Delta^{\leq k}\left(\right.$ Dyck $\left._{2}\right)$. In addition to th space required for computing the indices, $O(l)$ bits are required to store evaluation of $p_{x}$. But this does not need to be stored multiple times on the stack. Therefore, the algorithm uses $O(l+\sqrt{n \log n})=O(k \log n+\sqrt{n \log n}) \operatorname{space}$.

The proof of correctness and the error analysis are similar to the proof of Theorem 2 . Thus we get Theorem 4. The detailed algorithm is given below.

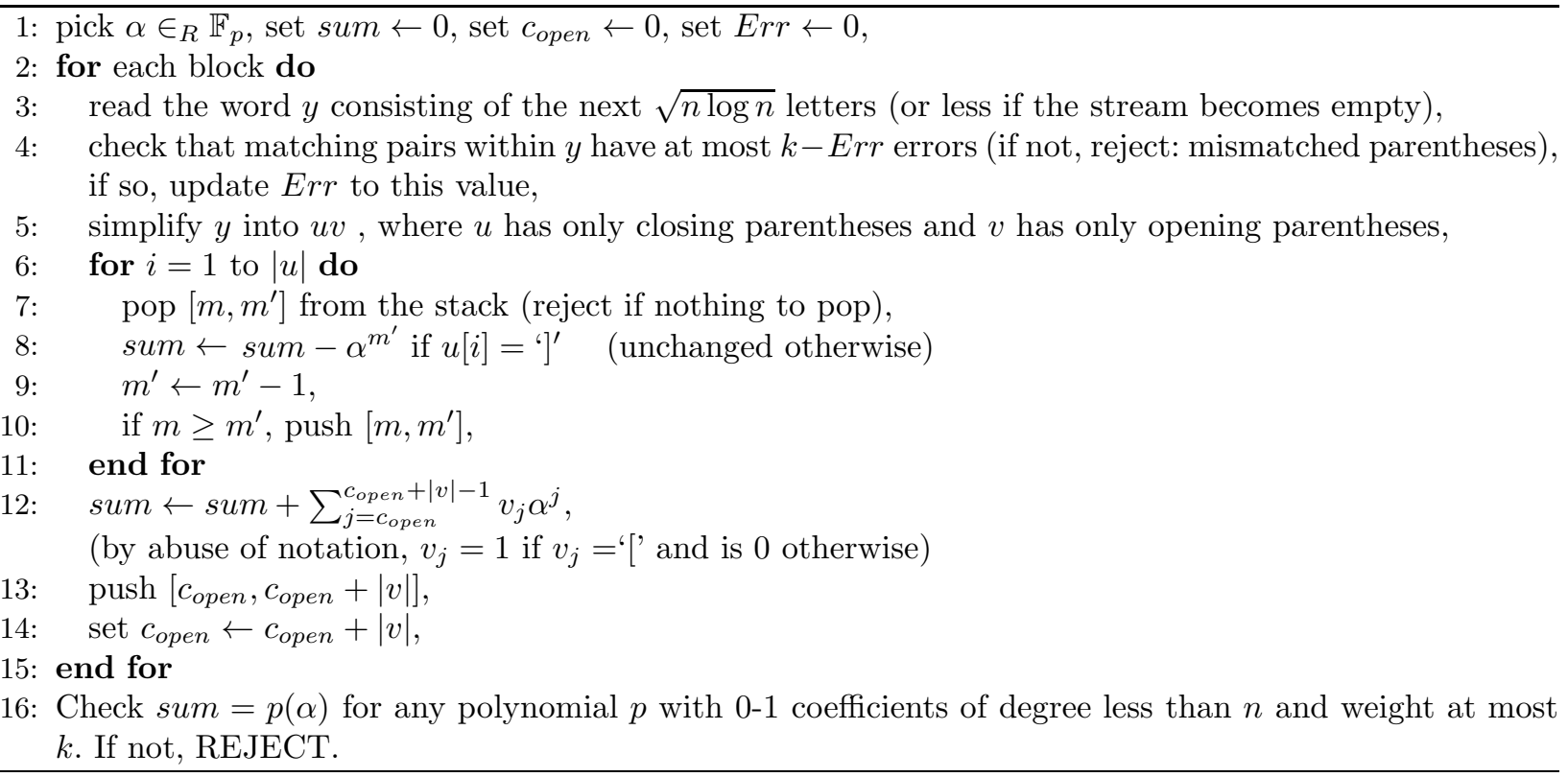

Reducing the randomness The ideas used in reducing randomness for $\Delta \leq k$ (1-turn-Dyck $\left.{ }_{2}\right)$ also work for reducing randomness in the membership testing of $\Delta^{\leq k}\left(\right.$ Dyck $\left._{2}\right)$. Here, instead of hashing the input positions, we hash the indices using the random hash functions. For computing the indices, we use the procedure described above. Instead of computing polynomials, we compute hashes and follow the steps as in Sections $3.1,3.2$, and 3.3.

We get the following theorem:

Theorem 5. For every constant $0 \leq \epsilon \leq 1$, there is randomized one-pass algorithm that tests membership in $\Delta^{\leq k}\left(\right.$ Dyck $\left._{2}\right)$ using $O\left(k^{1+\epsilon}+\sqrt{n \log n}\right)$ space, $O(\log n)$ randomness, $O\left(\log { }^{O(1)} n+k^{O(1)}\right)$ time and errs with probability $1 / 8$. 


\section{Lower bound}

We will show a lower bound for $\mathrm{PHam}_{n, k, k+2}$ by reducing the augmented indexing problem $\operatorname{IND}_{\mathcal{U}}^{a}$ (see [19) to it.

Let $\mathcal{U} \cup\{\perp\}$ denote a large input domain, where $\perp \notin \mathcal{U}$. Define the problem $\operatorname{IND}_{\mathcal{U}}^{a}$ as follows: Alice and Bob receive inputs $x=\left(x_{1}, x_{2}, \ldots, x_{N}\right) \in \mathcal{U}^{N}$ and $y=\left(y_{1}, y_{2}, \ldots, y_{N}\right) \in(U \cup\{\perp\})^{N}$, respectively. The inputs have the following promise: There is some unique $i \in[N]$, such that $y_{i} \in \mathcal{U}$, and for $k<i: x_{k}=y_{k}$, and for $k>i: y_{k}=\perp$. The problem $\operatorname{IND}_{\mathcal{U}}^{a}$ is defined only over such promise inputs and $\operatorname{IND}_{\mathcal{U}}^{a}(x, y)=1$ if and only if $x_{i}=y_{i}$.

In [19, Corollary 3.1] they proved the following result:

Theorem 6 ([19]). Any randomized one-way communication protocol that makes $\delta=1 / 4|\mathcal{U}|$ error requires $\Omega(N \log 1 / \delta)$ bits of communication.

We use this result and prove a lower bound for $\mathrm{PHam}_{n, k, k+2}$. follows:

Let $|\mathcal{U}|=n / k$. Let $f_{A}: \mathcal{U} \rightarrow\{0,1\}^{3 n / k}$, and $f_{B}:(\mathcal{U} \cup\{\perp\}) \rightarrow\{0,1\}^{\frac{3 n}{k}}$ be encoding functions defined as

$$
\begin{aligned}
& f_{A}: u_{i} \mapsto A_{1} A_{2} \ldots A_{n / k}, \text { where } A_{j}=\left\{\begin{array}{l}
110 \text { if } j=i \\
000 \text { otherwise }
\end{array}\right. \\
& f_{B}: u_{i} \mapsto B_{1} B_{2} \ldots B_{n / k}, \text { where } B_{j}=\left\{\begin{array}{l}
011 \text { if } j=i \\
000 \text { otherwise }
\end{array}\right. \\
& f_{B}(\perp)=0^{3 n / k} .
\end{aligned}
$$

On promise inputs $x, y \in \mathcal{U}^{k}$, let $F_{A}(x)$ and $F_{B}(y)$ be defined as $f_{A}\left(x_{1}\right) f_{A}\left(x_{2}\right) \ldots f_{A}\left(x_{k}\right)$ and $f_{B}\left(y_{1}\right) f_{B}\left(y_{2}\right) \ldots f_{B}\left(y_{k}\right)$, respectively.

Under this encoding, it is easy to see that $\mathrm{PHam}_{3 n, 2 k, 2 k+2}\left(F_{A}(x), F_{B}(y)\right)=1$ if and only if $\operatorname{IND}_{\mathcal{U}}^{a}(x, y)=1$. Suppose $i+1$ is the first position where $\perp$ appears in $y$. For each $j<i$ we have $x_{j}=y_{j}$ so the Hamming distance of $F_{A}\left(x_{j}\right)$ and $F_{B}\left(y_{j}\right)$ is 2 . Also for every position $j>i$ we have $y_{j}=\perp$ and hence $F_{B}\left(y_{j}\right)=0^{3 n / k}$, which results in a Hamming distance of 2 between $F_{A}\left(x_{j}\right)$ and $F_{B}\left(x_{j}\right)$. So the Hamming distance between $F_{A}(x)$ and $F_{B}(y)$ is $2(k-1)$ plus the Hamming distance between $f_{A}\left(x_{i}\right)$ and $f_{B}\left(y_{i}\right)$, which is 2 iff $x_{i}=y_{i}$ and 4 iff $x_{i} \neq y_{i}$ (since $\left.x_{i}, y_{i} \in \mathcal{U}\right)$.

Therefore we get the following lower bound:

Theorem 7 (Theorem 1 restated). Any randomized one-way protocol that makes at most $k / n$ error and computes $\mathrm{PHam}_{3 n, 2 k, 2 k+2}$, requires $\Omega(k \log (n / k))$ bits. In fact the lower bound holds for distinguishing between the case $\Delta(x, y)=2 k$ and $\Delta(x, y)=2 k+2$.

By Theorem 2 this bound is optimal when $n \geq k^{2}$ (and in fact when $n \geq k^{1+\epsilon}$, for constant $\epsilon>0$ ).

\section{References}

1. Alon, Goldreich, Hastad, and Peralta. Addendum to "simple construction of almost k-wise independent random variables". RSA: Random Structures 6 Algorithms, 4, 1993.

2. Noga Alon, Michael Krivelevich, Ilan Newman, and Mario Szegedy. Regular languages are testable with a constant number of queries. In SIAM Journal on Computing, pages 645-655, 1999.

3. Noga Alon, Yossi Matias, and Mario Szegedy. The space complexity of approximating the frequency moments. In Proceedings of the twenty-eighth annual ACM symposium on Theory of computing, STOC '96, pages 20-29, New York, NY, USA, 1996. ACM.

4. Ajesh Babu, Nutan Limaye, Jaikumar Radhakrishnan, and Girish Varma. Streaming algorithms for language recognition problems. Technical Report arXiv:1104.0848, Arxiv, 2011.

5. Ajesh Babu, Nutan Limaye, and Girish Varma. Streaming algorithms for some problems in log-space. In Jan Kratochvl, Angsheng Li, Jir Fiala, and Petr Kolman, editors, Theory and Applications of Models of Computation, volume 6108 of Lecture Notes in Computer Science, pages 94-104. Springer Berlin, Heidelberg, 2010. 10.1007/9783-642-13562-0-10. 
6. David A. Mix Barrington and James Corbett. On the relative complexity of some languages in $\mathrm{NC}^{1}$. Information Processing Letters, 32(5):251 - 256, 1989.

7. Feng Cao, Martin Ester, Weining Qian, and Aoying Zhou. Density-based clustering over an evolving data stream with noise. In In 2006 SIAM Conference on Data Mining, pages 328-339, 2006.

8. Amit Chakrabarti, Graham Cormode, Ranganath Kondapally, and Andrew McGregor. Information cost tradeoffs for augmented index and streaming language recognition. In Proceedings of the 2010 IEEE 51st Annual Symposium on Foundations of Computer Science, FOCS '10, pages 387-396, 2010.

9. Anindya De, Omid Etesami, Luca Trevisan, and Madhur Tulsiani. Improved pseudorandom generators for depth 2 circuits. In $A P P R O X-R A N D O M$, pages 504-517, 2010.

10. Sumit Ganguly. Data stream algorithms via expander graphs. In Proceedings of the 19th International Symposium on Algorithms and Computation, pages 52-63, 2008.

11. Wei Huang, Yaoyun Shi, Shengyu Zhang, and Yufan Zhu. The communication complexity of the hamming distance problem. Information Processing Letters, 99(4):149 - 153, 2006.

12. Rahul Jain and Ashwin Nayak. The space complexity of recognizing well-parenthesized expressions. Technical Report TR10-071, Electronic Colloquium on Computational Complexity, http://eccc.hpi-web.de/, April 19 2010. Revised July 5, 2010.

13. Eyal Kushilevitz and Noam Nisan. Communication Complexity. Cambridge University Press, New York, NY, USA, 2006.

14. Frédéric Magniez, Claire Mathieu, and Ashwin Nayak. Recognizing well-parenthesized expressions in the streaming model. In STOC, 2009.

15. S. Muthukrishnan. Data streams: Algorithms and applications. Foundations and Trends in Theoretical Computer Science, 1(2), 2005.

16. N. Nisan. Pseudorandom generators for space-bounded computations. In Proceedings of the twenty-second annual ACM symposium on Theory of computing, STOC '90, pages 204-212, New York, NY, USA, 1990. ACM.

17. Michal Parnas, Dana Ron, and Ronitt Rubinfeld. Testing parenthesis languages. In PROCEEDINGS OF THE 5 TH INTERNATIONAL WORKSHOP ON RANDOMIZATION AND APPROXIMATION TECHNIQUES IN COMPUTER SCIENCE, pages 261-272. Springer-Verlag, 2001.

18. Atri Rudra and Steve Uurtamo. Data stream algorithms for codeword testing. In ICALP (1), pages 629-640, 2010.

19. Jayram T. S. and Woodruff David. Optimal bounds for johnson-lindenstrauss transforms and streaming problems with sub-constant error. In SIAM: ACM-SIAM Symposium on Discrete Algorithms (SODA11), SODA '11, 2011.

20. Ronen Shaltiel. Weak derandomization of weak algorithms: Explicit versions of yao's lemma. Computational Complexity, Annual IEEE Conference on, 0:114-125, 2009.

21. Salil Vadhan. Pseudorandomness, 2010. Monograph in preparation for FnTTCS, available at http://people.seas.harvard.edu/ salil/pseudorandomness/

22. Andrew Chi-Chih Yao. On the power of quantum fingerprinting. In Proceedings of the thirty-fifth annual ACM symposium on Theory of computing, STOC '03, pages 77-81, 2003. 